\title{
Mood disorders in the elderly: prevalence, functional impact, and management challenges
}

This article was published in the following Dove Press journal:

Neuropsychiatric Disease and Treatment

24 August 2016

Number of times this article has been viewed

\author{
Leandro da Costa Lane \\ Valiengo' \\ Florindo Stella ${ }^{1,2}$ \\ Orestes Vicente Forlenza' \\ 'Laboratory of Neuroscience \\ (LIM-27), Department and Institute \\ of Psychiatry, Faculty of Medicine, \\ University of São Paulo, Sao Paulo, \\ ${ }^{2}$ Biosciences Institute, Universidade \\ Estadual Paulista, Rio Claro, Brazil
}

Correspondence: Orestes Vicente Forlenza

Laboratory of Neuroscience (LIM-27),

Instituto de Psiquiatria do Hospital

das Clínicas da Faculdade de Medicina

da USP, Rua Doutor Ovídio Pires

de Campos 785, 05403-0I0 São

Paulo, SP, Brazil

Tel +55 II $266 \mid 7283$

Fax +55 II $266 \mid 7535$

Email forlenza@usp.br

\begin{abstract}
Despite the lower prevalence of severe mood disorders in the elderly as compared to younger adults, late-life depression and bipolar disorder (BD) are more strongly associated with negative outcomes related to the presence of medical comorbidities, cognitive deficits, and increased suicide risk and overall mortality. The mechanisms that contribute to these associations are probably multifactorial, involving pathological factors related directly and indirectly to the disease itself, ranging from biological to psychosocial factors. Most of the accumulated knowledge on the nature of these associations derives from naturalistic and observational studies, and controlled data are still scarce. Nonetheless, there has clearly been a recent growth of the scientific interest on late-life BD and geriatric depression. In the present study, we review the most relevant studies on prevalence, clinical presentation, and cognitive/functional impact of mood disorders in elderly. Several clinical-epidemiological studies were dedicated to the study of the prevalence of mood disorders in old age in distinct settings; however, fewer studies investigated the underlying neurobiological findings and treatment specificities in late-life depression and $\mathrm{BD}$. In the present study, we further discuss the implications of these findings on the management of mood disorders in older adults.
\end{abstract}

Keywords: depression, bipolar disorder, psychogeriatric, geriatric, old age, older adults

\section{Introduction}

Mood disorders are frequent in old age and their prevalence is increasing with population aging. ${ }^{1}$ Because of its severe consequences, late-life mood disorders may be regarded as an important public health problem. Depression and bipolar disorder (BD) in the elderly are associated with medical comorbidities and cognitive decline, in addition to increased risk of dementia, suicide risk, and overall mortality. ${ }^{2-4}$ There are particularities in the etiology, clinical presentation, and management of mood disorders in older adults. Awareness of these differences and their clinical implications are important for the effective treatment of mood disorders in the elderly. Here, we present a review of current research on the epidemiology, neurobiology, and treatment of late-life mood disorders.

\section{Epidemiology of mood disorders in late life}

Mood disorders represent the most common source of psychiatric morbidity in older adults, including unipolar (depressive disorder) and bipolar (manic-depressive) subtypes, with varying degrees of severity (Table 1). Unipolar depression occurs in $10 \%-38 \%$ of the elderly population. ${ }^{2,5}$ Overall, $35.3 \%$ of the cases of late-life depression may be regarded as mild, $51.9 \%$ as moderate, and $12.7 \%$ as severe. ${ }^{5}$ Increasing age in depressed patients accounts for higher percentages of cases with unfavorable clinical course, with higher relapse rates, ${ }^{6}$ worse treatment response, and incomplete 
Table I Epidemiological aspects of mood disorders in the elderly

\begin{tabular}{lll}
\hline & BD & MDD \\
\hline Prevalence (\%) & $0.1-0.5$ & $10-38$ \\
Female (\%) & 66 & 52 \\
Cognitive deficit & Yes & Yes \\
Treatment-resistance & Yes & Yes \\
Under-recognized (\%) & - & 40 \\
\hline
\end{tabular}

Abbreviations: BD, bipolar disorder; MDD, major depression disorder.

functional recovery. ${ }^{7,8}$ Furthermore, depression in the elderly is a highly under-recognized syndrome $(40 \%-60 \%$ of cases). ${ }^{9}$ The prevalence of treatment-resistant depression is also common among the elderly, with an estimated rate of 26-41/100 person-years. $^{10}$

The 12-month prevalence rate of $\mathrm{BD}$ in patients 65 years of age or older is $\sim 0.1 \%-0.5 \% .{ }^{11-13}$ The highest lifetime prevalence rate is $1 \%$ among those aged 60 years or older. ${ }^{14}$ In spite of this relatively low prevalence of BD among elders living in the community, data from clinical settings suggest that late-life $\mathrm{BD}$ is a frequent diagnosis related to hospital admission at specialized services, representing $4 \%-8 \%$ of the inpatients at psychogeriatric units. ${ }^{15}$ Accordingly, a study about the patterns of mental health service use in the US indicated that up to $17 \%$ of the elderly patients presenting at emergency facilities had a diagnosis of BD. ${ }^{16}$ In a clinical study assessing elderly patients admitted to hospital care due to acute manic episodes, Benedetti et al ${ }^{17}$ found that the mean age of onset of $\mathrm{BD}$ in these patients was 49 years. Accordingly, Moorhead and Young ${ }^{18}$ found in a retrospective study that the age of onset of BD type I was also 49 years, in cases with no family history of the disorder. About half of all BD patients experience depression as their first major mood episode, and the time-lag until their first manic episode is $\sim 15$ years. ${ }^{19}$ In a large case-registry study conducted in Australia, Almeida and Fenner ${ }^{20}$ compared cases of early- and late-onset $\mathrm{BD}$ in the elderly and concluded that late-onset cases represented only $8 \%$ of all cases. In this study, earlier age of onset was associated with higher prevalence of mixed affective episodes. In addition, there seems to be an association between increased age at onset and duration of the affective episodes. ${ }^{21} \mathrm{In} \mathrm{BD}$, the risk of suicide is lowest for patients aged 35 years or older, ${ }^{22}$ but the rate of suicide among older patients has not been specifically investigated. ${ }^{1}$

\section{Clinical aspects of mood disorders in the elderly}

An older adult presenting with symptoms of mania, mixed states, or depression requires a careful differential diagnostic evaluation to exclude any organic disease and identify any potentially treatable medical conditions. ${ }^{23}$ Laboratory workup should include a comprehensive metabolic panel, complete blood cell count, thyroid function, toxicology screen, and more specialized assessments (as neuroimaging and specialized studies - electroencephalogram and lumbar puncture) as may be indicated by the history, physical, or neurological examination..$^{23}$

In older adults, depression may present with more sleep disturbance, fatigue, psychomotor retardation, and hopelessness about the future than younger adults with the same condition. ${ }^{24}$ Other very common presentations in elderly with depression are complaints of poor memory and concentration, slower cognitive processing speed, and executive dysfunction confounding with dementia, called pseudodementia. ${ }^{25} \mathrm{Neu}-$ rological comorbidities may be associated with depression in the elderly, for example, Parkinson's disease and stroke, which may account for differences in the clinical presentation of symptoms. ${ }^{26}$ Poststroke depression is frequently associated with severe vegetative symptoms. ${ }^{27}$ Depression in Parkinson's disease is usually milder and with less anhedonia than depression in otherwise healthy geriatric patients. ${ }^{26}$

The clinical differences between early- and late-onset BD in the elderly are small and most likely attributable to differences in the duration of illness. ${ }^{20}$ Older adults with early-onset $\mathrm{BD}$ more frequently present with mixed episodes, whereas late-onset $\mathrm{BD}$ elders display higher levels of premorbid psychosocial functional deficit, less severe psychopathology, and more cognitive impairment, particularly in psychomotor performance and mental flexibility. ${ }^{16,20,28}$

\section{Neurobiological correlates of geriatric depression and BD geriatric depression}

Growing evidence of neurobiological correlates of depression in late-life has received special attention from the scientific community, focusing particularly on neuroendocrine dysfunctions, neuroimaging, cerebrovascular correlates, neurotrophins, and inflammatory mechanisms, in addition to the association between major depression and risk of dementia, namely Alzheimer's disease (AD).

\section{Neuroendocrine dysfunctions}

Deregulation of the hypothalamic-pituitary-adrenal (HPA) axis function is associated with suicidal behavior and age-associated alterations in HPA axis and is a predictor of suicide risk in late-life depression. ${ }^{29}$ Genotypes associated with increased cortisol secretion and variants of angiotensin-converting enzyme gene are the risk factors for late-life depression. ${ }^{30}$ 


\section{Neuroimaging}

Structural and functional neuroimaging studies in geriatric major depression indicate a significant volume loss and metabolic changes in frontal cortical-subcortical pathways. ${ }^{31}$ A meta-analysis demonstrated the association of late-life depression with the reduction of hippocampal volume and with a large amount of atrophy in the precuneus, superior frontal gyrus, and ventromedial frontal cortex. ${ }^{32}$ Disordered connections affecting brain networks, such as default mode network, affective network, salience network, and the cognitive control network, have also been suggested in geriatric depression. ${ }^{33}$ Hyperactivity of the default mode network and disorganization of the affective network and other corticallimbic connections were recently documented, and such abnormalities were rescued back to normal standards upon successful antidepressant treatment. ${ }^{34}$

\section{Cerebrovascular disease}

The perforating arteries deliver the blood supply to the basal ganglia, a subcortical hub that is highly connected to prefrontal structures. When these vessels are damaged, connections from basal ganglia to prefrontal regions and other frontal structures are impaired, resulting in increased risk of depression and cognitive impairment, namely executive dysfunction, slowed mental processing speed, and diminished ability of insight..$^{35}$ According to the "vascular depression" hypothesis, white matter lesions disrupting axonal tracts in orbital and dorsolateral prefrontal regions, as well as in limbic cortical-subcortical pathways, have been associated with late-onset major depression; these pathways are critically involved in emotional regulation and decision-making strategies. ${ }^{35}$ Cerebrovascular disease has been considered as a central risk factor for the development of vascular depression; conversely, executive impairment was associated with increased risk of vascular dementia. ${ }^{36}$ This clinical picture has been designated as a depression-executive dysfunction syndrome characterized by psychomotor retardation, lack of interest, apathy, lack of initiative, impaired processing speed, more motivational problems, depressive ideation, limited insight, and prominent difficulty to perform daily instrumental activities. ${ }^{37}$

Therefore, disordered frontolimbic and frontostriatal connections may account for many of the clinical manifestations of vascular depression. ${ }^{36}$

Although there is considerable empiric support for the validity of a vascular depression subtype, fundamental questions remain open, including how the illness is defined, how vascular disease and depression influence each other, why it is not a progressive disorder, and whether white matter hypointensities and global vascular risk factors are responsible for poor outcome and poor response to antidepressive treatment. ${ }^{37}$

\section{Reduced neurotrophic support}

Given the potential correlates of neurotrophin changes with neuropsychiatric disorders, a recent study aimed to determine the peripheral concentration of nerve growth factor in patients with late-onset depression. ${ }^{38}$ In comparison to age- and sex-matched healthy controls, patients with lateonset depression - both when acutely depressed and also after remission through pharmacological treatment - had evidence of abnormal regulation of neurotrophic factors implicated in neuronal survival. It is yet unclear whether changes in peripheral nerve growth factor parallel those that occur in the central nervous system. However, it is known that peripheral alterations of certain neurotrophins, such as brain-derived neurotrophic factor, have been implicated in distinct brain dysfunctions, including late-onset depression. ${ }^{39}$ Reduced serum brain-derived neurotrophic factor levels may contribute to the physiopathology of late-life depression. ${ }^{40}$

\section{Inflammatory mechanisms}

An extensive review indicated that upregulation of proinflammatory cytokines in depressed patients may interfere with neurotransmission, particularly in certain limbic structures, such as the amygdala, hippocampus, and nucleus accumbens, with unfavorable impacts on emotional regulation..$^{41}$ Accordingly, a meta-analysis revealed a compensatory inflammatory response with higher concentrations of tumor necrosis factor- $\alpha$ (TNF- $\alpha$ ) and interleukin (IL)- 6 in patients with major depression. ${ }^{42}$ Taken together, such studies indicate that the severity of depression symptoms bears a positive correlation with inflammatory response and an inverse association with neurotrophic support.

Other biological mechanisms have been implicated in major depression in older adults, such as reduced serum concentration of glial cell-line-derived neurotrophic factor, ${ }^{43}$ decreased serum levels of adiponectin with potential negative impact on cognitive functions, ${ }^{44}$ increased serum concentration of soluble TNF receptor $2,{ }^{45}$ and higher IL- $1 \beta$ levels. ${ }^{46}$ These changes may contribute to proinflammatory state related to physiopathology of depression in the elderly.

\section{Cognitive impairment}

Cognitive symptoms are irrefutably present in geriatric depression; yet, studies have not reached a consensus regarding the pattern of neuropsychological impairment in these patients, or whether the observed deficits during an acute 
depressive episode will remain (and for how long) after symptom remission. Nonetheless, cognitive impairment has been consistently documented in patients with acute depressive symptoms, ${ }^{47}$ as well as among those with long-lasting depressive disorder. ${ }^{48}$ In the latter condition, persistent cognitive impairment was documented in the remission phase, particularly involving the ability to perform tasks demanding visual information search. ${ }^{48}$ Several longitudinal studies have suggested that cognitive changes may persist after remission of the depressive episode. With the aim to examine cognition and social functioning in remitted depression, a prospective study investigated a sample of patients from the general population who fulfilled clinical criteria for major depressive episode; persistent cognitive impairment was detected after 3 years of follow-up, in spite of symptomatic remission and reestablishment of social functioning. ${ }^{49}$

Changes in cognition comprise distinct neuropsychological domains involving frontal processes, such as executive functions, problem solving and planning, mental flexibility, decision-making ability, and inhibitory control; also, selective and sustained attention, working memory, and semantic or phonemic verbal fluency have been reported in depressed patients. ${ }^{48}$

\section{Cognitive changes and risk of dementia}

Cumulative evidence supports that major depression increases the risk of dementia, including $\mathrm{AD}$, particularly when the affective symptoms are accompanied by consistent subjective memory complaints or by actual cognitive impairment. Although subjective memory complaints were associated with depression in people without dementia, ${ }^{50}$ approaches to evaluation of these complaints are very general and still devoid of precise assessment strategies. Nonetheless, patients with concomitant major depression and mild cognitive impairment may have impaired performance on instrumental activities of the daily living, which are associated with deficits in executive functions, verbal fluency, episodic memory, sustained attention, working memory, and processing speed. ${ }^{51}$

Chronic depression during the life course increases the risk of dementia, and when the disease begins in late life, depression may reflect a prodromal stage of dementia, including $\mathrm{AD} .{ }^{52}$ Barnes et $\mathrm{al}^{52}$ examined the association between depressive symptoms and dementia in a long-term longitudinal study of 13,535 individuals. The study included individuals who volunteered to this cohort 4 decades earlier and were first assessed at the age of 40-45 years. Baseline data were retrieved, and participants reassessed at a mean age of 81 years, subclassified according to the occurrence of depression in the period (no depressive symptoms, depressive symptoms in mid-life only, depressive symptoms in late life only, or depressive symptoms in mid-life and late life), and followed up for an additional period of 6 years. Dementia was detected among $20.7 \%$ of the patients with no depressive symptoms, $23.5 \%$ of those with depressive symptoms in midlife, and $31.5 \%$ of those with depression in late life. ${ }^{52}$

A single photon emission computed tomography study was conducted by Cho et $\mathrm{al}^{53}$ to address brain perfusion patterns in patients with depression and cognitive impairment, compared to AD patients and healthy controls. The former patients had evidence of reduced cerebral blood flow in temporal and parietal regions, in a similar pattern as observed among AD patients, whereas cognitively unimpaired depressed patients displayed a distinct perfusion pattern with reduced blood flow in frontal areas. ${ }^{53}$ Diffusion tensor imaging studies further suggest that patients with depression tend to display abnormalities in the connectivity between cortical-limbic structures (eg, from amygdala to dorsolateral prefrontal cortex) with reduced performance on executive tasks. ${ }^{54}$

\section{Depression and AD biomarkers}

Molecular imaging studies with positron emission tomography (PET) indicating increased amyloid- $\beta$ (A $\beta)$ deposition in the brain of depressed elders reinforced the association between major depression and the risk for AD. Using 18-florbetapir, Chung et $\mathrm{al}^{55}$ showed an association between lifetime depression and $\mathrm{A} \beta$ accumulation in the frontal cortex of patients with amnestic mild cognitive impairment. In a longitudinal study with a mean follow-up time of 6.2 years, Qiu et $\mathrm{al}^{56}$ analyzed the risk of AD in nondemented depressed individuals, addressing plasma concentrations of $A \beta$ and the apolipoprotein E genotype; the authors found a positive association between the risk of dementia and plasma $A \beta$ levels, especially among those carrying the apoE*4 allele.

\section{Bipolar disorder \\ Cognitive changes and risk of dementia in late-life BD}

Older adults with BD display a widespread array of cognitive symptoms, which may be critical during acute phases of abnormal mood states but may persist (in a lesser extent) through euthymia. Presumably these cognitive changes reflect the long-lasting charge from multiple disease-related neurobiological mechanisms. ${ }^{57}$ The most frequently reported cognitive deficits are related to executive dysfunction, affecting abstract thinking, mental set-shifting, inhibitory control, 
decision-making ability and verbal fluency, in addition to impairments in sustained attention, psychomotor abilities, and verbal memory. ${ }^{57}$ These changes invariably exert a negative impact on functionality and psychosocial performance. Impairment of occupational activities may be seen in $30 \%-60 \%$ of older BD patients. ${ }^{58}$

Table 2 summarizes the most frequently reported cognitive abnormalities in geriatric $\mathrm{BD}$ and major depression. Cognitive impairment and dementia are frequent long-term outcomes of BD and, therefore, particularly affect patients at older ages. The precise nature of the pathological process that ultimately leads to dementia in BD is not yet fully understood and will be discussed in the next section below.

\section{Neurobiological mechanisms related to dementia in $\mathrm{BD}$}

Dementia in BD can be regarded as secondary to the cumulative burden of the disease itself (clinical severity and treatment-related adverse events), in addition to the presence of medical and neurological comorbidities (particularly cerebrovascular). However, it is difficult to rule out the participation of intrinsic biological mechanisms that ultimately upregulate intracellular signals related to neurodegeneration. Postmortem studies indicate that histopathological changes are very subtle and may at least in part arise from the effect of chronic medication use; however, certain changes may be detected prior to treatment, and the fact that long-term treatment does modify the natural course of the disease renders the notion of $\mathrm{BD}$ as a neurodegenerative disease difficult to support. ${ }^{59}$ Lifetime use of antipsychotics, antidepressants, anticonvulsants, and particularly lithium - the latter drug associated with neuroprotective properties in the recent

Table 2 Frequent cognitive abnormalities in older patients with MDD and BD

\begin{tabular}{llc}
\hline Affected cognitive abilities & MDD & BD \\
\hline Executive functions & + & + \\
$\quad$ Abstract thinking & & + \\
$\quad$ Cognitive set-shifting & & + \\
Inhibitory control & & + \\
$\quad$ Decision-making ability & + & + \\
Working memory & + & \\
Sustained attention & + & \\
Verbal fluency & + & + \\
Episodic memory & + & + \\
Processing speed & & \\
Psychomotor skills & & \\
\hline
\end{tabular}

Note: + , affected.

Abbreviations: BD, bipolar disorder; MDD, major depression disorder. literature ${ }^{60}$ - may change not only the psychopathology but also the biological markers of the disease. ${ }^{59}$

The course of cognitive changes in BD that frequently lead to impairments in middle age and ultimately to dementia in late life, is clearly different from that observed in primary neurodegenerative dementia, namely AD, which is another indication of a distinct etiology. Two recent studies conducted in our group demonstrated that older, cognitively impaired, BD patients do not display a significant effect of the apolipoprotein $\mathrm{E}^{*} 4$ allele ${ }^{61}$ or the pathological markers of $\mathrm{AD}$ in the cerebrospinal fluid" ${ }^{62}$ - the so-called "pathological signature" of AD in the cerebrospinal fluid, which is defined by reduced concentrations of the $A \beta$ peptide along with increased total Tau and phosphorylated Tau. ${ }^{62,63}$ These findings suggest that the dementing process in $\mathrm{BD}$ follows a distinct biological route compared to $\mathrm{AD}$, which is compatible with the distinct pattern observed in clinical progression of neuropsychological deficits. Therefore, one may hypothesize that persistent and progressive cognitive deterioration in $\mathrm{BD}$ reflects the long-time outcome of the disease, presumably sharing mechanisms of cognitive decline with other major psychiatric disorders. ${ }^{64}$

\section{Structural and functional neuroimaging}

Although converging efforts point toward elucidating the neurobiological basis of mental disorders, structural neuroimaging techniques have so far provided insufficient support to distinguish BD from other psychiatric illnesses or to efficiently separate mania from depression or healthy controls. ${ }^{65}$ Studies have described structural abnormalities of hippocampus, subgenual prefrontal cortex, anterior cingulate, temporo-parietal regions, thalamus, basal ganglia, and cortico-subcortical pathways, among others engaged in cognition processes. ${ }^{66}$

In a recent cross-sectional study in $\mathrm{BD}$ type 1 , Cao et $\mathrm{al}^{67}$ examined verbal memory and hippocampal volume at late stages of the disease (defined according to the number of manic episodes and hospitalizations); results indicated that memory loss was associated with reduced hippocampal volume in patients with severe forms of BD (ie, ten or more manic episodes and at least one hospitalization due to acute mania or depression), suggesting that hippocampal atrophy may be a late marker of the disease in the brain. Another study by Abé et $\mathrm{a}^{68}$ examined cortical thickness, volume, and surface area in BD patients types I and II; compared to healthy controls, BD patients had lower cortical thickness in several structures, including frontal regions, anterior cingulate cortex, temporal regions, insula, and medial occipital lobe. 
A functional neuroimaging study based on ${ }^{18} \mathrm{~F}$-fluoro deoxyglucose uptake (fluorodeoxyglucose positron emission tomography) provided evidence of deregulation of corticolimbic glucose metabolism in BD, which was characterized by hypoactivation of prefrontal cortex and hyperactivation of anterior limbic regions, in a distinct pattern compared to that observed in patients with schizophrenia. ${ }^{69}$

The investigation of microstructural abnormalities also constitutes a promising approach in the investigation of the neurobiology of dementia in BD. According to a comprehensive review of postmortem studies, dendritic atrophy and loss of glial cells were detected mainly in medial prefrontal cortex in $\mathrm{BD},{ }^{59}$ explaining at least in part the microstructural abnormalities of connections between prefrontal regions and the limbic system, and compatible with previous evidence of reduced fractional anisotropy measured by diffusion tensor imaging. ${ }^{59,65}$

\section{Inflammatory mechanisms}

Investigations at cellular level provide consistent evidence on dysfunction of biological mechanisms in BD, including proinflammatory overactivity of microglia system, which in turn changes the HPA axis. ${ }^{65}$ A comprehensive review on $\mathrm{BD}$ reported that increased proinflammatory cytokines are closely related to dysfunction of the HPA, volumetric reduction, and hypoactivation of frontal lobes. ${ }^{70}$ The authors also documented disorders of serotonin and dopamine neurotransmitters in amygdala, hippocampus, and nucleus accumbens - brain areas crucially implicated in the regulation of emotional manifestations, reward processes, and psychomotor behavior. $^{70}$

\section{Lithium and neuroprotection}

There is convergent evidence on the clinical benefits of lithium treatment in BD and mood disorders. However, more recently, cumulative data from experimental models and a few clinical explorations further supported the notion that lithium therapy may also upregulate neurotrophic and neuroprotective cascades. A case-control study conducted by Nunes et $\mathrm{al}^{71}$ compared older BD patients chronically treated with lithium with a similar group of patients treated with other mood-stabilizing drugs (nonusers of lithium). The authors detected a lower prevalence of dementia (supposedly AD) among lithium-treated patients compared to nonusers ( $5 \% \mathrm{vs}$ $33 \% ; P<0.001$ ), suggesting a reduction of the risk of dementia to similar levels as those reported for the general elderly population. These findings were further corroborated by other authors with a distinct methodological approach. ${ }^{72,73}$
Individuals chronically exposed to lithium as the main treatment for $\mathrm{BD}$ also presented increased cortical thickness and volume, specifically in medial occipital areas, when compared with nonusers of lithium. ${ }^{68}$ These data are in agreement with other studies supporting the hypothesis that lithium may also have neurotrophic proprieties, in addition to its well-accepted mood-stabilizing effect. ${ }^{74}$ In addition, lithium may specifically modify pathological processes implicated in AD. ${ }^{75}$ The mechanism of action could be attributed to the fact that lithium inhibits both the expression ${ }^{76}$ and activity $^{77}$ of the enzyme glycogen synthase kinase- $3 \beta,{ }^{71}$ as well as the inhibition of the overproduction of $A \beta$ and the hyperphosphorylation of tau protein, ${ }^{78}$ all directly involved in the neurodegenerative process in AD.

Other putative effects attributed to lithium involve neuroprotection mechanisms, eg, stimulation of synthesis and release of neurotrophins, with increased resilience against neurotoxic insults, and stimulation of hippocampal neurogenesis. ${ }^{79}$ In addition, lithium may modulate neuronal plasticity, including neurogenesis, synaptic pruning, and synaptic maturation, transcriptional control, modulation of oxidative stress, and decreasing of inflammatory processes; other mechanisms are related to the reduction of IL- $1 \beta$ and TNF- $\alpha{ }^{79-81}$

\section{Treatment aspects of geriatric depression and BD}

General guidelines for the treatment of geriatric depression are largely similar to the ones accepted for the treatment of middle-aged adults. Randomized clinical trials with depressed older adults have demonstrated similar efficacy for selective serotonin reuptake inhibitors, dual inhibitors, tricyclic antidepressants, and monoamine oxidase inhibitors, with moderate to large effect sizes. ${ }^{26}$ However, some types of antidepressants are better for conditions associated with old age. ${ }^{82}$ Older patients usually have more comorbidities and use more medications. Because of that, antidepressants with a better pharmacokinetic profile (ie, less prone to induce drug-drug interactions) should be considered as first choice: sertraline, citalopram, escitalopram, and desvenlafaxine. ${ }^{83}$ Moreover, selective serotonin reuptake inhibitors are not associated with anticholinergic and sedative effects, which is advantageous for the treatment of elderly patients.

Several modalities of psychotherapy demonstrated efficacy for treating depression in old age, including behavioral therapy, cognitive behavioral therapy, cognitive bibliotherapy, problem-solving therapy, brief psychodynamic therapy, and life review therapy. ${ }^{26}$ Electroconvulsive therapy (ECT) is used more often in older adults than in any other age group 
yielding improvement in $>80 \%$ of patients in most trials; however, certain adverse events, such as cardiac complications, memory loss, and delirium, demand caution in the use of ECT for the treatment of depression in older adults. ${ }^{84}$ Cognitive effects are transient in most but not all cases. ${ }^{84}$

There are no randomized clinical trials for the treatment of old-age BD. The available literature is largely represented by uncontrolled, open label, or exploratory trials of larger mixed-age populations studies. This topic was subdivided to address the particular aspects of the treatment of depression, mania, hypomania, and mixed episodes.

\section{Bipolar depression}

Several uncontrolled studies have shown a decrease in depressive symptoms with the use of aripiprazole and asenapina for old-age BD. ${ }^{85,86}$ However, older adults are very sensitive to drug-related effects such as extrapyramidal symptom. ${ }^{1}$

One open-label trial evaluated the efficacy of lamotrigine in 57 late-onset BD patients. ${ }^{23}$ They received add-on lamotrigine to the regular treatment with the response and remission rates of $64.8 \%$ and $57.4 \%$, respectively, with a mean lamotrigine dose of $150.90 \mathrm{mg} / \mathrm{d}$. An exploratory analysis of two double-blind, randomized, placebo-controlled studies in bipolar depression compared quetiapine with placebo in mixed-age patients. In a subgroup of 72 patients aged 55-65 years, remission occurred more often with quetiapine (300 mg/d [45\%] and $600 \mathrm{mg} / \mathrm{d}$ [48\%]) than placebo $28 \%{ }^{1}$ The use of lithium and valproate in this group of patients is not well established. The literature on bipolar depression is at best represented by a few retrospective studies suggesting improvement of depressive symptoms. ${ }^{87-89}$ There are limited data about the use of ECT in bipolar depression in old-age, restricted to small case series and extrapolations from mixedage samples. ${ }^{1}$

\section{Acute mania, hypomania, and mixed episodes}

Lithium salts are largely the preferred choice for the treatment of acute mania in old-age BD..$^{90}$ Lithium may reduce suicide risk and also the risk of dementia. ${ }^{73}$ Several open-label studies have demonstrated efficacy of lithium in the treatment of acute mania. ${ }^{1}$ Valproate has also been suggested to be effective in old-age BD for the treatment of mania. ${ }^{91} \mathrm{In}$ a retrospective report, the efficacy of lithium compared to valproate for the treatment of acute mania was similar, with response rates of $82 \%$ and $75 \%$, respectively. ${ }^{92}$

Some studies demonstrated a possible role for the use of antipsychotics in this group of patients. An exploratory analysis of a mixed-age test group treated with olanzapine indicated good response in patients older than 50 years. ${ }^{1}$ A randomized, placebo-controlled, trial on a mixed-age BD sample reported efficacy of quetiapine in the subsample of patients aged 59 years and older. ${ }^{93}$

\section{Maintenance therapy}

A secondary analysis of a study conducted by Sajatovic et al ${ }^{94}$ in a sample of 86 older BD patients indicated efficacy of lamotrigine in delaying relapse of depression, whereas lithium was more effective in delaying relapse of manic symptoms. Another randomized, open-label study comparing lithium to divalproex in a mixed-age BD sample showed that lithium monotherapy or lithium in combination with valproate was superior to valproate alone in delaying relapse..$^{95}$

\section{Other treatments}

Repetitive transcranial magnetic stimulation, a technique in which rapidly changing magnetic fields are used to induce electrical currents in the brain, is a promising intervention for depression with a treatment effect size as large as the effect size seen when using antidepressant medications for depression..$^{96}$ One study reviewed the available trials of repetitive transcranial magnetic stimulation for the treatment of depression in older patients and found only four well-designed, randomized clinical trials conducted so far..$^{97}$ The intervention seems to be well tolerated and safe. Two of these trials demonstrated better response in the active group compared to sham, and two trials did not find any differences between groups.

Physical exercise may also yield benefits as a complementary treatment for depression in older adults. One controlled study proposed that exercise training may be as effective as antidepressants and better than placebo. ${ }^{98}$ Benefits may also apply to refractory depression, with further evidence of reducing relapse rate over as long as 5 years for those who continued exercising. ${ }^{98,99}$

\section{Conclusion}

Along with population aging, many age-related conditions (mood disorders included) steadily increase over time, necessitating the need for specific diagnostic tools and treatment approaches. Unipolar depression and BD are chronic and frequently severe illnesses that impair functionality, reduce quality of life, and increase mortality. There is still limited evidence on how to specifically deal with mood disorders in late life, particularly considering the high rate of comorbidities and association with neurocognitive and degenerative outcomes. 


\section{Acknowledgment}

We thank: the Fundação de Amparo à Pesquisa de São Paulo (grant no 2011/19892-3), Associação Beneficente Alzira Denise Hertzog da Silva, and JNK Empreendimentos e Incorporações, all of whom funded this study.

\section{Disclosure}

The authors report no conflicts of interest in this work.

\section{References}

1. Sajatovic M, Strejilevich SA, Gildengers AG, et al. A report on older-age bipolar disorder from the International Society for Bipolar Disorders Task Force. Bipolar Disord. 2015;17(7):689-704.

2. Blazer DG. Depression in late life: review and commentary. J Gerontol A Biol Sci Med Sci. 2003;58(3):249-265.

3. Brailean A, Comijs HC, Aartsen MJ, et al. Late-life depression symptom dimensions and cognitive functioning in the Longitudinal Aging Study Amsterdam (LASA). J Affect Disord. 2016;201:171-178.

4. Mirza SS, Wolters FJ, Swanson SA, et al. 10-year trajectories of depressive symptoms and risk of dementia: a population-based study. Lancet Psychiatry. 2016;3(7):628-635.

5. Guerra M, Prina AM, Ferri CP, et al. A comparative cross-cultural study of the prevalence of late life depression in low and middle income countries. J Affect Disord. 2016;190:362-368.

6. Mitchell AJ, Subramaniam H. Prognosis of depression in old age compared to middle age: a systematic review of comparative studies. Am J Psychiatry. 2005;162(9):1588-1601.

7. Tedeschini E, Levkovitz Y, Iovieno N, Ameral VE, Nelson JC, Papakostas GI. Efficacy of antidepressants for late-life depression: a meta-analysis and meta-regression of placebo-controlled randomized trials. J Clin Psychiatry. 2011;72(12):1660-1668.

8. Beekman AT, Penninx BW, Deeg DJ, de Beurs E, Geerling SW, van Tilburg W. The impact of depression on the well-being, disability and use of services in older adults: a longitudinal perspective. Acta Psychiatr Scand. 2002;105(1):20-27.

9. Alamo C, López-Muñoz F, García-García P, García-Ramos S. Riskbenefit analysis of antidepressant drug treatment in the elderly. Psychogeriatrics. 2014;14(4):261-268.

10. Little JT, Reynolds CF 3rd, Dew MA, et al. How common is resistance to treatment in recurrent, nonpsychotic geriatric depression? Am J Psychiatry. 1998;155(8):1035-1038.

11. Weissman MM, Leaf PJ, Tischler GL, et al. Affective disorders in five United States communities. Psychol Med. 1988;18(1):141-153.

12. Hirschfeld RM, Calabrese JR, Weissman MM, et al. Screening for bipolar disorder in the community. J Clin Psychiatry. 2003;64(1): $53-59$.

13. Unützer J, Simon G, Pabiniak C, Bond K, Katon W. The treated prevalence of bipolar disorder in a large staff-model HMO. Psychiatr Serv. 1998;49(8):1072-1078.

14. Kessler RC, Berglund P, Demler O, Jin R, Merikangas KR, Walters EE. Lifetime prevalence and age-of-onset distributions of DSM-IV disorders in the National Comorbidity Survey Replication. Arch Gen Psychiatry. 2005;62(6):593-602.

15. Yassa R, Nair V, Nastase C, Camille Y, Belzile L. Prevalence of bipolar disorder in a psychogeriatric population. J Affect Disord. 1988;14(3): 197-201.

16. Depp CA, Lindamer LA, Folsom DP, et al. Differences in clinical features and mental health service use in bipolar disorder across the lifespan. Am J Geriatr Psychiatry. 2005;13(4):290-298.

17. Benedetti A, Scarpellini P, Casamassima F, et al. Bipolar disorder in late life: clinical characteristics in a sample of older adults admitted for manic episode. Clin Pract Epidemiol Ment Health. 2008;4:22.

18. Moorhead SR, Young AH. Evidence for a late onset bipolar-I disorder sub-group from 50 years. $J$ Affect Disord. 2003;73(3):271-277.
19. Shulman KI, Tohen M, Satlin A, Mallya G, Kalunian D. Mania compared with unipolar depression in old age. Am J Psychiatry. 1992;149(3): 341-345.

20. Almeida OP, Fenner S. Bipolar disorder: similarities and differences between patients with illness onset before and after 65 years of age. Int Psychogeriatr. 2002;14(3):311-322.

21. Young RC. Bipolar mood disorders in the elderly. Psychiatr Clin North Am. 1997;20(1):121-136.

22. Tsai SY, Kuo CJ, Chen CC, Lee HC. Risk factors for completed suicide in bipolar disorder. J Clin Psychiatry. 2002;63(6):469-476.

23. Sajatovic M, Chen P. Geriatric bipolar disorder. Psychiatr Clin North Am. 2011;34(2):319-333, vii.

24. Christensen H, Jorm AF, Mackinnon AJ, et al. Age differences in depression and anxiety symptoms: a structural equation modelling analysis of data from a general population sample. Psychol Med. 1999; 29(2):325-339.

25. Butters MA, Whyte EM, Nebes RD, et al. The nature and determinants of neuropsychological functioning in late-life depression. Arch Gen Psychiatry. 2004;61(6):587-595.

26. Fiske A, Wetherell JL, Gatz M. Depression in older adults. Anпи Rev Clin Psychol. 2009;5:363-389.

27. Paradiso S, Vaidya J, Tranel D, Kosier T, Robinson RG. Nondysphoric depression following stroke. J Neuropsychiatry Clin Neurosci. 2008;20(1):52-61.

28. Azorin JM, Kaladjian A, Adida M, Fakra E. Late-onset bipolar illness: the geriatric bipolar type VI. CNS Neurosci Ther. 2012;18(3):208-213.

29. Jokinen J, Nordström P. HPA axis hyperactivity as suicide predictor in elderly mood disorder inpatients. Psychoneuroendocrinology. 2008;33(10):1387-1393.

30. Ancelin ML, Carrière I, Scali J, Ritchie K, Chaudieu I, Ryan J. Angiotensin-converting enzyme gene variants are associated with both cortisol secretion and late-life depression. Transl Psychiatry. 2013;3:e322.

31. Sexton CE, Mackay CE, Ebmeier KP. A systematic review and metaanalysis of magnetic resonance imaging studies in late-life depression. Am J Geriatr Psychiatry. 2013;21(2):184-195.

32. Boccia M, Acierno M, Piccardi L. Neuroanatomy of Alzheimer's disease and late-life depression: a coordinate-based meta-analysis of MRI studies. J Alzheimers Dis. 2015;46(4):963-970.

33. Dutta A, McKie S, Deakin JF. Resting state networks in major depressive disorder. Psychiatry Res. 2014;224(3):139-151.

34. Gudayol-Ferré E, Peró-Cebollero M, González-Garrido AA, GuàrdiaOlmos J. Changes in brain connectivity related to the treatment of depression measured through fMRI: a systematic review. Front Hum Neurosci. 2015;9:582

35. Alexopoulos GS, Meyers BS, Young RC, Campbell S, Silbersweig D, Charlson M. 'Vascular depression' hypothesis. Arch Gen Psychiatry. 1997;54(10):915-922.

36. Alexopoulos GS. The vascular depression hypothesis: 10 years later. Biol Psychiatry. 2006;60(12):1304-1305.

37. Jellinger K. The enigma of vascular depression. Austin Alzheimers J Parkinsons Dis. 2016;3:1026.

38. Diniz BS, Teixeira AL, Machado-Vieira R, Talib LL, Gattaz WF, Forlenza OV. Reduced serum nerve growth factor in patients with late-life depression. Am J Geriatr Psychiatry. 2013;21(5):493-496.

39. Duman RS, Monteggia LM. A neurotrophic model for stress-related mood disorders. Biol Psychiatry. 2006;59(12):1116-1127.

40. Diniz BS, Teixeira AL, Talib LL, Mendonça VA, Gattaz WF, Forlenza OV. Serum brain-derived neurotrophic factor level is reduced in antidepressant-free patients with late-life depression. World J Biol Psychiatry. 2010;11(3):550-555.

41. Zunszain PA, Anacker C, Cattaneo A, Carvalho LA, Pariante CM. Glucocorticoids, cytokines and brain abnormalities in depression. Prog Neuropsychopharmacol Biol Psychiatry. 2011;35(3):722-729.

42. Dowlati Y, Herrmann N, Swardfager W, et al. A meta-analysis of cytokines in major depression. Biol Psychiatry. 2010;67(5):446-457.

43. Diniz BS, Teixeira AL, Miranda AS, Talib LL, Gattaz WF, Forlenza OV. Circulating Glial-derived neurotrophic factor is reduced in late-life depression. J Psychiatr Res. 2012;46(1):135-139. 
44. Diniz BS, Teixeira AL, Campos AC, et al. Reduced serum levels of adiponectin in elderly patients with major depression. J Psychiatr Res. 2012;46(8):1081-1085.

45. Diniz BS, Teixeira AL, Talib LL, Mendonça VA, Gattaz WF, Forlenza OV. Increased soluble TNF receptor 2 in antidepressant-free patients with late-life depression. J Psychiatr Res. 2010;44(14):917-920.

46. Diniz BS, Teixeira AL, Talib L, Gattaz WF, Forlenza OV. Interleukin1beta serum levels is increased in antidepressant-free elderly depressed patients. Am J Geriatr Psychiatry. 2010;18(2):172-176.

47. Castaneda AE, Suvisaari J, Marttunen M, et al. Cognitive functioning in a population-based sample of young adults with a history of nonpsychotic unipolar depressive disorders without psychiatric comorbidity. J Affect Disord. 2008;110(1-2):36-45.

48. Hammar A, Lund A, Hugdahl K. Long-lasting cognitive impairment in unipolar major depression: a 6-month follow-up study. Psychiatry Res. 2003;118(2):189-196.

49. Airaksinen E, Wahlin A, Larsson M, Forsell Y. Cognitive and social functioning in recovery from depression: results from a populationbased three-year follow-up. J Affect Disord. 2006;96(1-2):107-110.

50. Balash Y, Mordechovich M, Shabtai H, Giladi N, Gurevich T, Korczyn AD. Subjective memory complaints in elders: depression, anxiety, or cognitive decline? Acta Neurol Scand. 2013;127(5):344-350.

51. Koehler M, Kliegel M, Wiese B, et al. Malperformance in verbal fluency and delayed recall as cognitive risk factors for impairment in instrumental activities of daily living. Dement Geriatr Cogn Disord. 2011;31(1): 81-88.

52. Barnes DE, Yaffe K, Byers AL, McCormick M, Schaefer C, Whitmer RA. Midlife vs late-life depressive symptoms and risk of dementia: differential effects for Alzheimer disease and vascular dementia. Arch Gen Psychiatry. 2012;69(5):493-498.

53. Cho MJ, Lyoo IK, Lee DW, et al. Brain single photon emission computed tomography findings in depressive pseudodementia patients. J Affect Disord. 2002;69(1-3):159-166.

54. Thomas EJ, Elliott R. Brain imaging correlates of cognitive impairment in depression. Front Hum Neurosci. 2009;3:30.

55. Chung JK, Plitman E, Nakajima S, et al. Lifetime history of depression predicts increased amyloid- $\beta$ accumulation in patients with mild cognitive impairment. J Alzheimers Dis. 2015;45(3):907-919.

56. Qiu WQ, Zhu H, Dean M, et al. Amyloid-associated depression and ApoE4 allele: longitudinal follow-up for the development of Alzheimer's disease. Int J Geriatr Psychiatry. 2016;31(3):316-322.

57. Forlenza OV, Aprahamian I. Cognitive impairment and dementia in bipolar disorder. Front Biosci (Elite Ed). 2013;5:258-265.

58. MacQueen GM, Young LT, Galway TM, Joffe RT. Backward masking task performance in stable, euthymic out-patients with bipolar disorder. Psychol Med. 2001;31(7):1269-1277.

59. Savitz JB, Price JL, Drevets WC. Neuropathological and neuromorphometric abnormalities in bipolar disorder: view from the medial prefrontal cortical network. Neurosci Biobehav Rev. 2014;42:132-147.

60. Diniz BS, Machado-Vieira R, Forlenza OV. Lithium and neuroprotection: translational evidence and implications for the treatment of neuropsychiatric disorders. Neuropsychiatr Dis Treat. 2013;9:493-500.

61. Kerr DS, Stella F, Radanovic M, Aprahamian I, Bertollucci PH, Forlenza OV. Apolipoprotein E genotype is not associated with cognitive impairment in older adults with bipolar disorder. Bipolar Disord. 2016; 18(1):71-77.

62. Forlenza OV, Aprahamian I, Radanovic M, et al. Cognitive impairment in late-life bipolar disorder is not associated with Alzheimer's disease pathological signature in the cerebrospinal fluid. Bipolar Disord. 2016;18(1):63-70.

63. Forlenza OV, Diniz BS, Gattaz WF. Diagnosis and biomarkers of predementia in Alzheimer's disease. BMC Med. 2010;8:89.

64. Soeiro-de-Souza MG, Dias VV, Figueira ML, et al. Translating neurotrophic and cellular plasticity: from pathophysiology to improved therapeutics for bipolar disorder. Acta Psychiatr Scand. 2012;126(5): 332-341.

65. Maletic V, Raison C. Integrated neurobiology of bipolar disorder. Front Psychiatry. 2014;5:98
66. Langan C, McDonald C. Neurobiological trait abnormalities in bipolar disorder. Mol Psychiatry. 2009;14(9):833-846.

67. Cao B, Passos IC, Mwangi B, et al. Hippocampal volume and verbal memory performance in late-stage bipolar disorder. J Psychiatr Res. 2016;73:102-107.

68. Abé C, Ekman CJ, Sellgren C, Petrovic P, Ingvar M, Landén M. Cortical thickness, volume and surface area in patients with bipolar disorder types I and II. J Psychiatry Neurosci. 2015;41(4):240-250.

69. Altamura AC, Bertoldo A, Marotta G, et al. White matter metabolism differentiates schizophrenia and bipolar disorder: a preliminary PET study. Psychiatry Res. 2013;214(3):410-414.

70. Barbosa IG, Bauer ME, Machado-Vieira R, Teixeira AL. Cytokines in bipolar disorder: paving the way for neuroprogression. Neural Plast. 2014;2014:360481.

71. Nunes PV, Forlenza OV, Gattaz WF. Lithium and risk for Alzheimer's disease in elderly patients with bipolar disorder. Br J Psychiatry. 2007; 190:359-360.

72. Kessing LV, Forman JL, Andersen PK. Does lithium protect against dementia? Bipolar Disord. 2010;12(1):87-94.

73. Kessing LV, Søndergård L, Forman JL, Andersen PK. Lithium treatment and risk of dementia. Arch Gen Psychiatry. 2008;65(11):1331-1335.

74. Machado-Vieira R, Manji HK, Zarate CA. The role of lithium in the treatment of bipolar disorder: convergent evidence for neurotrophic effects as a unifying hypothesis. Bipolar Disord. 2009;11(suppl 2): 92-109.

75. Forlenza OV, Diniz BS, Radanovic M, Santos FS, Talib LL, Gattaz WF Disease-modifying properties of long-term lithium treatment for amnestic mild cognitive impairment: randomised controlled trial. $\mathrm{Br} J$ Psychiatry. 2011;198(5):351-356.

76. Mendes CT, Mury FB, de Sá Moreira E, et al. Lithium reduces Gsk3b mRNA levels: implications for Alzheimer Disease. Eur Arch Psychiatry Clin Neurosci. 2009;259(1):16-22.

77. Ladeira RB, Joaquim HP, Talib LL, Nunes PV, Forlenza OV. Higher proportion of inactive Gsk3 $\beta$ in platelets of elderly patients with bipolar disorder: an effect of treatment? Rev Bras Psiquiatr. 2013;35(3): 274-278.

78. Klein PS, Melton DA. A molecular mechanism for the effect of lithium on development. Proc Natl Acad Sci U S A. 1996;93(16):8455-8459.

79. Diniz BS, Teixeira AL. Brain-derived neurotrophic factor and Alzheimer's disease: physiopathology and beyond. Neuromolecular Med. 2011;13(4):217-222.

80. Pasquali L, Busceti CL, Fulceri F, Paparelli A, Fornai F. Intracellular pathways underlying the effects of lithium. Behav Pharmacol. 2010; 21(5-6):473-492.

81. Forlenza OV, De-Paula VJ, Diniz BS. Neuroprotective effects of lithium: implications for the treatment of Alzheimer's disease and related neurodegenerative disorders. ACS Chem Neurosci. 2014;5(6):443-450.

82. Forlenza OV, Almeida OP, Stoppe A Jr, Hirata ES, Ferreira RCR. Antidepressant efficacy and safety of low-dose sertraline and standard-dose imipramine for the treatment of depression in older adults: results from a double-blind, randomized, controlled clinical trial. Int Psychogeriatr. 2001;13(1):75-84

83. Spina E, Santoro V, D'Arrigo C. Clinically relevant pharmacokinetic drug interactions with second-generation antidepressants: an update. Clin Ther. 2008;30(7):1206-1227.

84. Kelly KG, Zisselman M. Update on electroconvulsive therapy (ECT) in older adults. J Am Geriatr Soc. 2000;48(5):560-566.

85. Sajatovic M, Coconcea N, Ignacio RV, et al. Aripiprazole therapy in 20 older adults with bipolar disorder: a 12-week, open-label trial. J Clin Psychiatry. 2008;69(1):41-46.

86. Sajatovic M, Tatsuoka C, Dines P, et al. Patient choice as a driver of medication-switching in non-adherent individuals with bipolar disorder. Patient Prefer Adherence. 2014;8:487-491.

87. Abou-Saleh MT. Platelet MAO, personality and response to lithium prophylaxis. J Affect Disord. 1983;5(1):55-65.

88. Lepkifker E, Iancu I, Horesh N, Strous RD, Kotler M. Lithium therapy for unipolar and bipolar depression among the middle-aged and older adult patient subpopulation. Depress Anxiety. 2007;24(8):571-576. 
89. Sharma SD, Iqbal M. Lithium induced toxicity in rats: a hematological, biochemical and histopathological study. Biol Pharm Bull. 2005; 28(5):834-837.

90. D’Souza R, Rajji TK, Mulsant BH, Pollock BG. Use of lithium in the treatment of bipolar disorder in late-life. Curr Psychiatry Rep. 2011; 13(6):488-492.

91. Sajatovic M. Treatment of bipolar disorder in older adults. Int J Geriatr Psychiatry. 2002;17(9):865-873.

92. Chen B, Wang JF, Hill BC, Young LT. Lithium and valproate differentially regulate brain regional expression of phosphorylated CREB and c-Fos. Brain Res Mol Brain Res. 1999;70(1):45-53.

93. Sajatovic M, Mullen JA, Sweitzer DE. Efficacy of quetiapine and risperidone against depressive symptoms in outpatients with psychosis. J Clin Psychiatry. 2002;63(12):1156-1163.

94. Sajatovic M, Gyulai L, Calabrese JR, et al. Maintenance treatment outcomes in older patients with bipolar I disorder. Am J Geriatr Psychiatry. 2005;13(4):305-311.
95. BALANCE Investigators and Collaborators; Geddes JR, Goodwin GM, et al. Lithium plus valproate combination therapy versus monotherapy for relapse prevention in bipolar I disorder (BALANCE): a randomised open-label trial. Lancet. 2010;375(9712):385-395.

96. Aleman A, Slotema CW, Sommer IE. rTMS deserves a fair chance as a novel treatment for depression. Acta Psychiatr Scand. 2014;130(5): 324-325.

97. Sabesan P, Lankappa S, Khalifa N, Krishnan V, Gandhi R, Palaniyappan L. Transcranial magnetic stimulation for geriatric depression: promises and pitfalls. World J Psychiatry. 2015;5(2):170-181.

98. Barbour KA, Blumenthal JA. Exercise training and depression in older adults. Neurobiol Aging. 2005;26(suppl 1):119-123.

99. Motl RW, Konopack JF, McAuley E, Elavsky S, Jerome GJ, Marquez DX. Depressive symptoms among older adults: longterm reduction after a physical activity intervention. J Behav Med. 2005;28(4):385-394.

\section{Publish your work in this journal}

Neuropsychiatric Disease and Treatment is an international, peerreviewed journal of clinical therapeutics and pharmacology focusing on concise rapid reporting of clinical or pre-clinical studies on a range of neuropsychiatric and neurological disorders. This journal is indexed on PubMed Central, the 'PsycINFO' database and CAS, and is the official journal of The International Neuropsychiatric Association (INA). The manuscript management system is completely online and includes a very quick and fair peer-review system, which is all easy to use. Visit http://www.dovepress.com/testimonials.php to read real quotes from published authors. 